\title{
Elaboração e validação de escala diagramática para quantificação da mancha de isariopsis da videira
}

\author{
Elaboration and validation of a diagrammatic scale to quantify isariopsis leaf spot on grape
}

\author{
Giuvan Lenz $^{I^{*}}$ Ivan Dressler da Costa ${ }^{\mathrm{I}}$ Ricardo Silveiro Balardin ${ }^{\mathrm{I}}$ Leandro Nascimento Marques $^{\mathrm{I}}$ \\ Adriano Arrué ${ }^{\mathrm{I}}$ Maurício Silva Stefanelo' Carla Rejane Zemolin ${ }^{\mathrm{I}}$
}

\section{RESUMO}

A falta de um método-padrão de quantificação visual para doenças foliares pode levar a estimativas imprecisas da severidade destas, provocando conclusões erradas. O objetivo deste trabalho foi desenvolver uma escala diagramática para avaliação da mancha de isariopsis em videira causada por Isariopsis clavispora. Uma escala diagramática com seis níveis $(1,60 ; 3,20 ; 6,60 ; 13,00 ; 24,10$ e 40,24\%) foi desenvolvida e validada para quantificar a severidade da mancha de isariopsis em videira. A validação da escala foi realizada por oito avaliadores, que estimaram a severidade de 30 folhas com diferentes intensidades de doença. Por meio de regressão linear, confrontando valores de severidade reais e estimados, foram analisadas a acurácia e a precisão dos avaliadores que utilizaram a escala diagramática. Foram constadas acurácia e precisão das estimativas visuais efetuadas com o auxílio da escala diagramática. O coeficiente de determinação das regressões foi em média superior a 0,89, $e$ os erros absolutos foram concentrados dentro de limites aceitáveis. Os níveis de acurácia, precisão e reprodutibilidade das mensurações da severidade da mancha de isariopsis da videira melhoraram com o uso da escala diagramática. A escala proposta mostrou-se adequada para avaliação da severidade da mancha de isariopsis da videira.

Palavras-chave: Isariopsis clavispora, Pseudocercospora vitis, diagnose, mancha foliar, fitopatometria.

\section{ABSTRACT}

The lack of a standardized visual method may lead to inaccurate severity estimative and wrong conclusions. The objective of this research was to develop a diagrammatic scale to assess the severity of grape isariopsis leaf spot on grape, caused by Isariopsis clavispora. A diagrammatic scale with six levels of disease severity $(1.60 ; 3.20 ; 6.60 ; 13.00$; 24.10 e $40.24 \%$ ) was developed and validated to assess grape isariopsis leaf spot. For validation, the severity of 30 leaves presenting different intensities of the disease was estimated by eight raters. Accuracy and precision were determined by linear regression and by the actual severity scale. This one provided good levels of accuracy and accuracy of visual estimation. The coefficient determination average was higher than 0.89, and absolute errors were lower than acceptable limits. The levels of accuracy, precision and reproducibility measurements of isariopsis leaf spot severity on grape significantly improved with the use of diagrammatic scale. The proposed diagrammatic scale proved to be adequate for severity assessments of grape isariopsis leaf spot.

Key words: Isariopsis clavispora, Pseudocercospora vitis, diagnosis, leaf spot, phytopatometry.

\section{INTRODUÇÃO}

Em regiões quentes e úmidas, a mancha de isariopsis, causada por Isariopsis clavispora, assume grande importância devido à desfolha precoce das plantas que pode provocar, podendo prejudicar a maturação dos ramos e enfraquecer a planta para o ciclo seguinte (ZEMOLIN et al., 2006). Os sintomas se manifestam principalmente nas folhas, onde são bastante característicos. No limbo foliar, aparecem manchas bem definidas de contorno irregular e coloração inicialmente castanho-avermelhada, que mais tarde tornam-se escuras. As manchas podem atingir até 2cm de diâmetro, que podem coalescer com a evolução da doença. Apresentam um halo amarelado ou verde claro bem visível e, na face oposta da folha, no tecido correspondente, observa-se uma coloração pardacenta, que são as estruturas reprodutivas do

'Departamento de Defesa Fitossanitária, Centro de Ciências Rurais (CCR), Universidade Federal de Santa Maria (UFSM), 97105900, Santa Maria, RS, Brasil. E-mail: giuvanlenz@gmail.com.*Autor para correspondência. 
fungo. Essas estruturas desenvolvem-se, tanto na face superior, como na inferior da folha, e sob o ataque severo da doença provoca a queda prematura das folhas e a redução do vigor por falta de reservas que seriam acumuladas após a colheita da uva, prejudicando a maturação dos ramos e predispondo-os ao ataque de pragas e doenças (SONEGO \& GARRIDO, 2003).

A doença tem grande importância para cultivares americanas e híbridas, e as variedades européias apresentam resistência ao patógeno. Normalmente os tratamentos recomendados para antracnose e míldio são suficientes para controlar a mancha de isariopsis (AMORIM \& KUNIYUKI, 1997).

No manejo integrado de doenças, a quantificação das doenças de plantas assume grande importância. Porém, em alguns casos, é de difícil execução por ser um procedimento trabalhoso e relativamente oneroso para o produtor. Os dados sobre quantificação da incidência e severidade de doenças são importantes no manejo destas no sentido de avaliar diferentes medidas de controle cultural, biológico, resistência varietal e teste da eficácia de produtos fitossanitários (GOMES et al., 2004).

Apesar da subjetividade a que está sujeita, a avaliação visual da severidade deve fornecer dados acurados, precisos e reproduzíveis. A utilização de escalas diagramáticas pode reduzir a subjetividade das estimativas de severidade entre os avaliadores, melhorando a acurácia e precisão da avaliação (MARTINS et al., 2004; AQUINO et al., 2008; LENZ et al., 2009). No entanto, as escalas têm que ser reproduzíveis e devem apresentar níveis suficientes para caracterização do avanço no progresso da doença (GODOY et al., 2006).

No caso de doenças foliares, a severidade é a variável mais utilizada, e a sua avaliação é, normalmente, feita de forma subjetiva por meio de análises visuais e, consequentemente, as escalas diagramáticas tornam-se ferramentas fundamentais em tais estudos (GODOY et al., 1997).

A presença de certo nível de erro absoluto observado nas mensurações de intensidade de doença pode ser compensada pela rapidez e padronização quando utilizam-se escalas diagramáticas (STONEHOUSE, 1994). NUTTER \& WORAWITLIKIT (1989) afirmam que, para que um avaliador possa ser considerado excelente, o erro de suas estimativas deve estar dentro de um intervalo de $\pm 5 \%$ do valor real e, para ser considerado bom, o erro não pode ultrapassar $\pm 10 \%$.

O uso da escala permite ao avaliador situar sua estimativa num intervalo menor, compreendido entre dois dos níveis representados por seus diagramas
(AMORIM, 1995). Dessa forma, as escalas diagramáticas contribuem na redução do erro na estimativa visual da severidade de doenças. As escalas diagramáticas devem ser simples, aplicáveis em diferentes condições e ter intervalos suficientes para representar os diferentes níveis de severidade de uma determinada doença. Na elaboração de uma escala diagramática, alguns aspectos devem ser considerados, como as quantidades máximas de doença observadas no campo e representadas na escala, a representação dos sintomas da maneira mais próxima possível da realidade e os níveis de severidade, respeitando as limitações da acuidade da vista humana definidas pelas leis de estímulo-resposta de Weber e de Fechner, também conhecidas como lei de Weber-Fechner (HORSFALL \& BARRAT, 1945; NUTTER \& SCHULTZ, 1995).

Após a elaboração, as escalas devem ser testadas por diferentes indivíduos a fim de comprovar sua eficiência na estimativa da severidade. A precisão, medida de confiabilidade e/ou repetibilidade nas avaliações de doença, pode ser quantificada pelo coeficiente de determinação das equações de regressão linear estabelecidas entre a severidade real e as estimativas da severidade de uma determinada amostra. A precisão será tanto maior quanto mais próximo de 1,0 for o valor do coeficiente de determinação $\left(R^{2}\right)$. A acurácia, representada pelo grau de proximidade entre a estimativa média e a realidade, pode ser quantificada pelo coeficiente angular e pela interseção de linhas de regressão estabelecidas entre a severidade real e as estimativas da severidade de uma determinada amostra. A acurácia será tanto maior quanto mais próximo de 1,0 for o coeficiente angular e mais próxima de zero for a interseção.

Assim, considerando a necessidade do estabelecimento de um método com mínima subjetividade na avaliação da intensidade de perda de área foliar, foi estabelecido o presente trabalho com os objetivos de desenvolver e validar uma escala diagramática para avaliação da severidade da mancha de isariopsis da videira.

\section{MATERIAL E MÉTODOS}

Elaboração da escala diagramática

Para elaboração da escala diagramática, foram coletadas 100 folhas de plantas de videira, de diversas cultivares, com diferentes níveis de severidade de Isariopsis clavispora. A determinação da área foliar total e da área necrótica foi feita com o auxílio de um integrador de área foliar da marca LICOR ${ }^{\circledR}$ (Licor1000), por meio da separação do tecido necrótico da 
folha com auxílio de bisturi e posterior passagem no integrador. A severidade da doença foi calculada com base na percentagem de área foliar necrótica sob a área total da folha. Utilizando o valor máximo de severidade da doença constatada nas folhas coletadas, os intervalos da escala foram calculados com o auxílio do programa 2LOG ${ }^{\circledR}$ (TOVAR-SOTO et al., 2002).

Baseando-se na lei de Weber-Fechner de acuidade visual (HORSFALL \& COWLING, 1978), bem como na forma, distribuição e frequência das lesões, foi confeccionada, com a ajuda do editor gráfico CorelDRAW ${ }^{\circledR}$ (Corel), uma escala diagramática logarítmica com seis níveis de severidade (Figura 1) e nela representados os sintomas de cada nível, procurando-se manter os padrões de distribuição das lesões.

Validação da escala diagramática

Para validação da escala diagramática proposta, foram tomadas 30 folhas com diferentes níveis de severidade e apresentadas para oito avaliadores sem experiência prévia na avaliação de doenças, que estimaram a severidade da mancha de isariopsis da videira em uma primeira etapa sem e, posteriormente, com a escala diagramática. Equações de regressão linear entre a severidade real (variável independente) e a estimada (variável dependente) foram calculadas para cada avaliador (Figura 2). A acurácia das estimativas de cada avaliador foi determinada pelo teste $t$ aplicado às estimativas dos parâmetros: (1) de interseção da regressão linear $(a)$, para verificar a hipótese Ho: $a=0$; (2) do coeficiente angular da reta (b), para testar a hipótese Ho: $b=1$, ao nível de probabilidade $P=0,05$. A precisão foi estimada pelo coeficiente de determinação da equação de regressão $\left(R^{2}\right)$ e pela variação dos resíduos expressa pela severidade estimada menos a severidade real (Figuras 3 e 4).

\section{RESULTADOS E DISCUSSÃO}

Na validação da escala diagramática, todos os avaliadores mostraram-se com boa acurácia, pois os valores estimados de severidade ficaram próximos dos valores da severidade real. Conforme BERGAMIN FILHO \& AMORIM (1996), valores próximos entre valores estimados e valores reais determinam a acurácia das avaliações.

Quando a severidade da doença foi estimada sem o auxílio da escala diagramática, os avaliadores foram pouco precisos, com coeficientes de determinação variando entre 0,34 e 0,73 para as equações de regressão estabelecidas entre a severidade real e a estimada (Tabela 1). Por outro lado, quando a severidade da doença foi estimada com o auxílio da escala diagramática, os avaliadores se mostraram com precisão bem superior à observada sem o uso da escala,

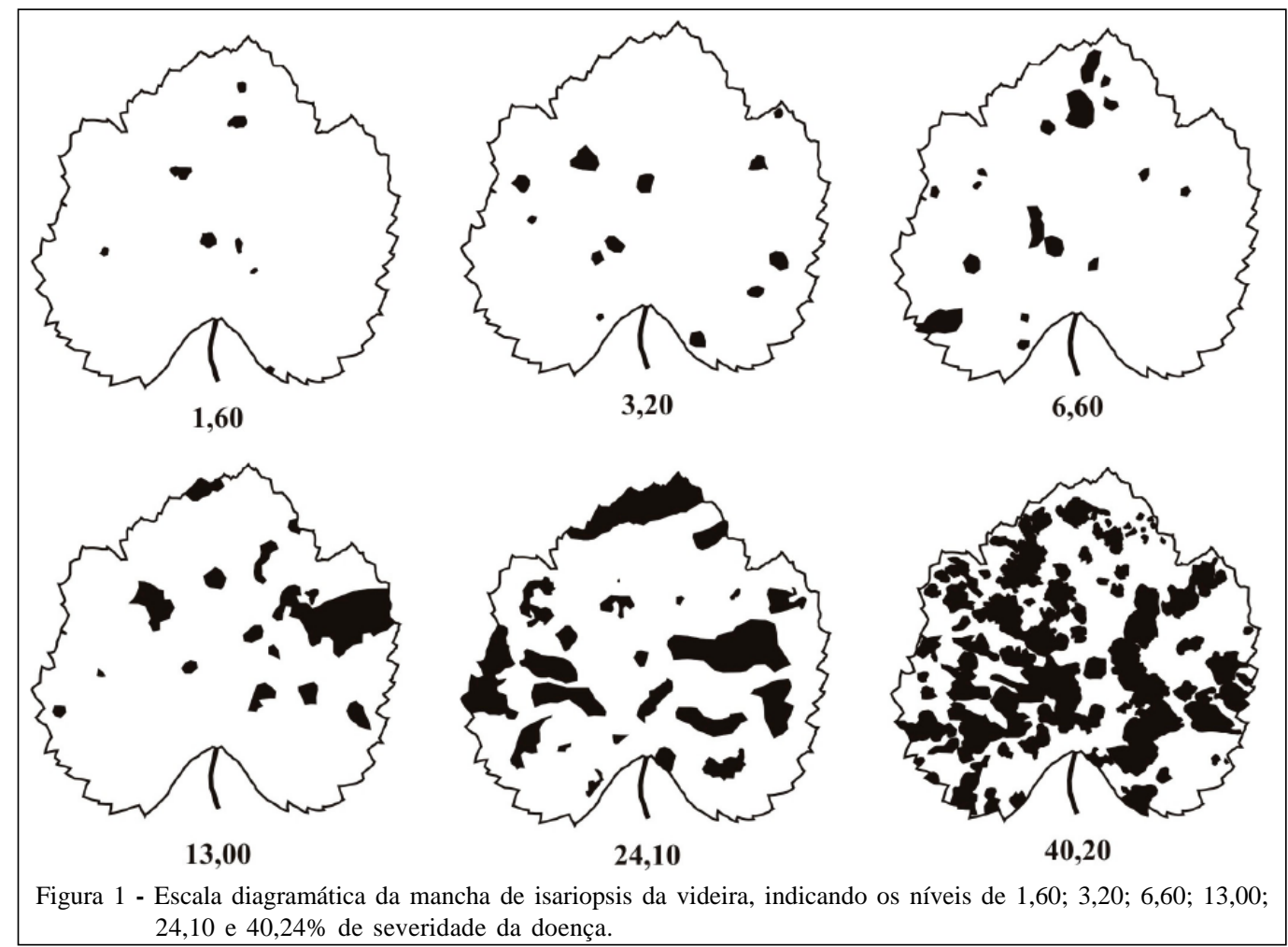

Ciência Rural, v.39, n.8, nov, 2009. 


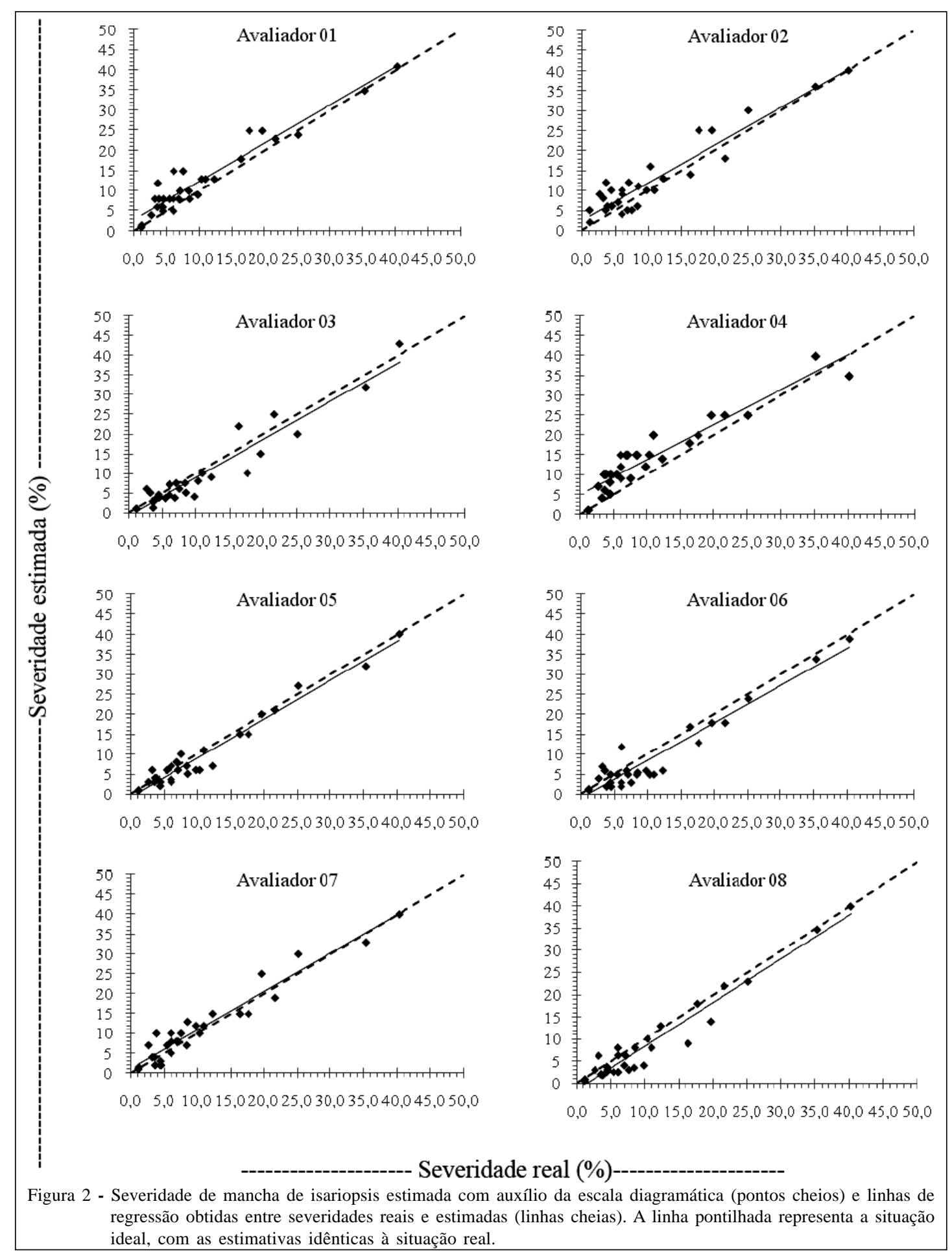

com coeficientes de determinação variando entre 0,89 e 0,96. Essa melhoria também tem sido verificada com a utilização de outras escalas diagramáticas (NASCIMENTO et al., 2005; LENZ et al., 2009), o que demonstra a importância dessa ferramenta em estudos de quantificação de doenças.
A precisão, conforme BERGAMIN FILHO \& AMORIM (1996), é um fator a ser considerado na validação de uma escala diagramática e é definida como a exatidão de uma operação em que há rigor ou refinamento na medida. A precisão pode ser avaliada 


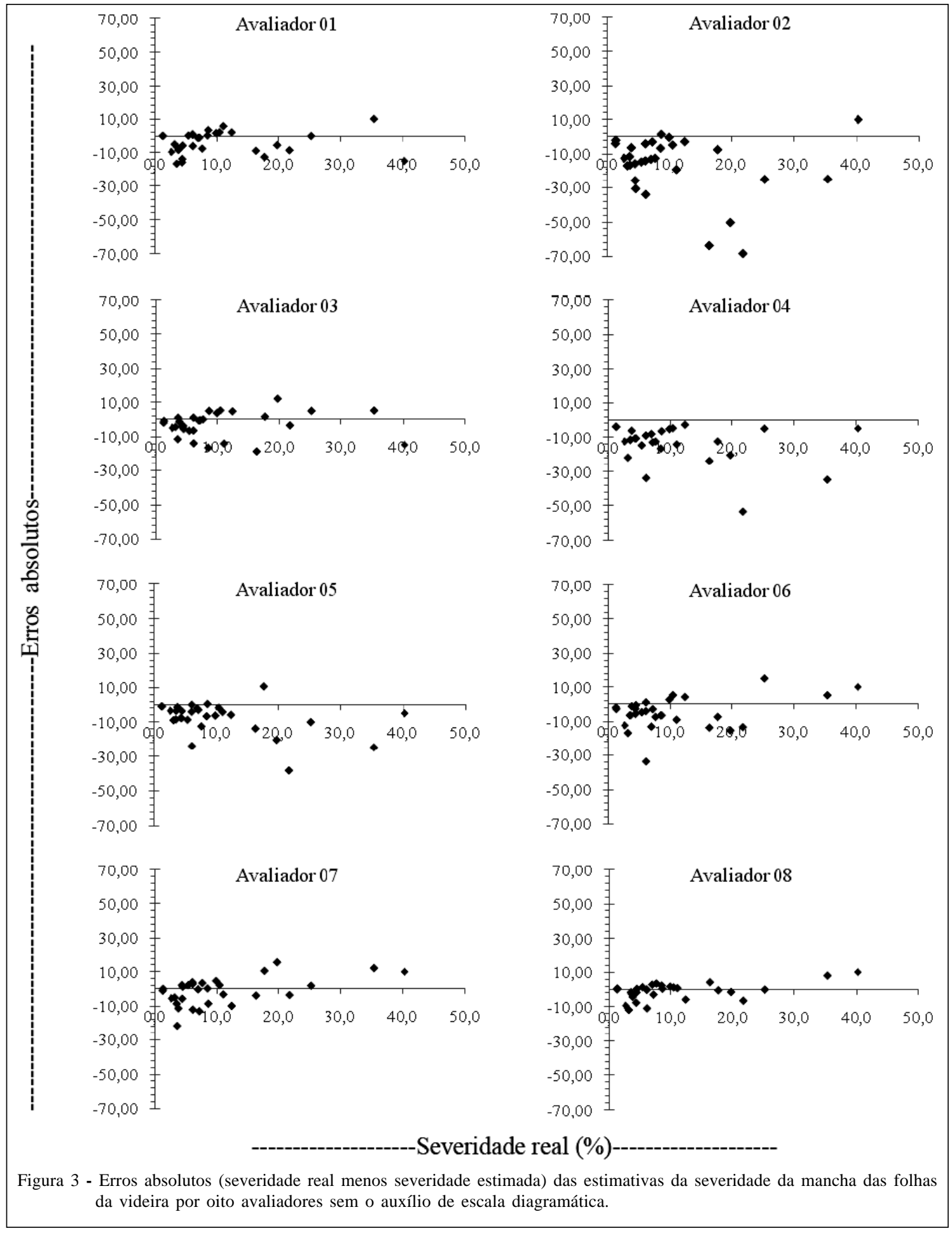

por meio do coeficiente de determinação da regressão, que deve ser próximo de um, e pela variação dos erros absolutos expressa pela diferença entre severidade estimada e real.

A média geral das estimativas de severidade da doença de todos os avaliadores ficou situada em
16,88\% quando estes não utilizaram a escala e 10,95\%, quando utilizaram a escala diagramática. Portanto, considerando que a média da severidade real foi de $10,54 \%$, a utilização da escala fez com que os avaliadores estimassem a severidade com maior precisão.

Ciência Rural, v.39, n.8, nov, 2009. 


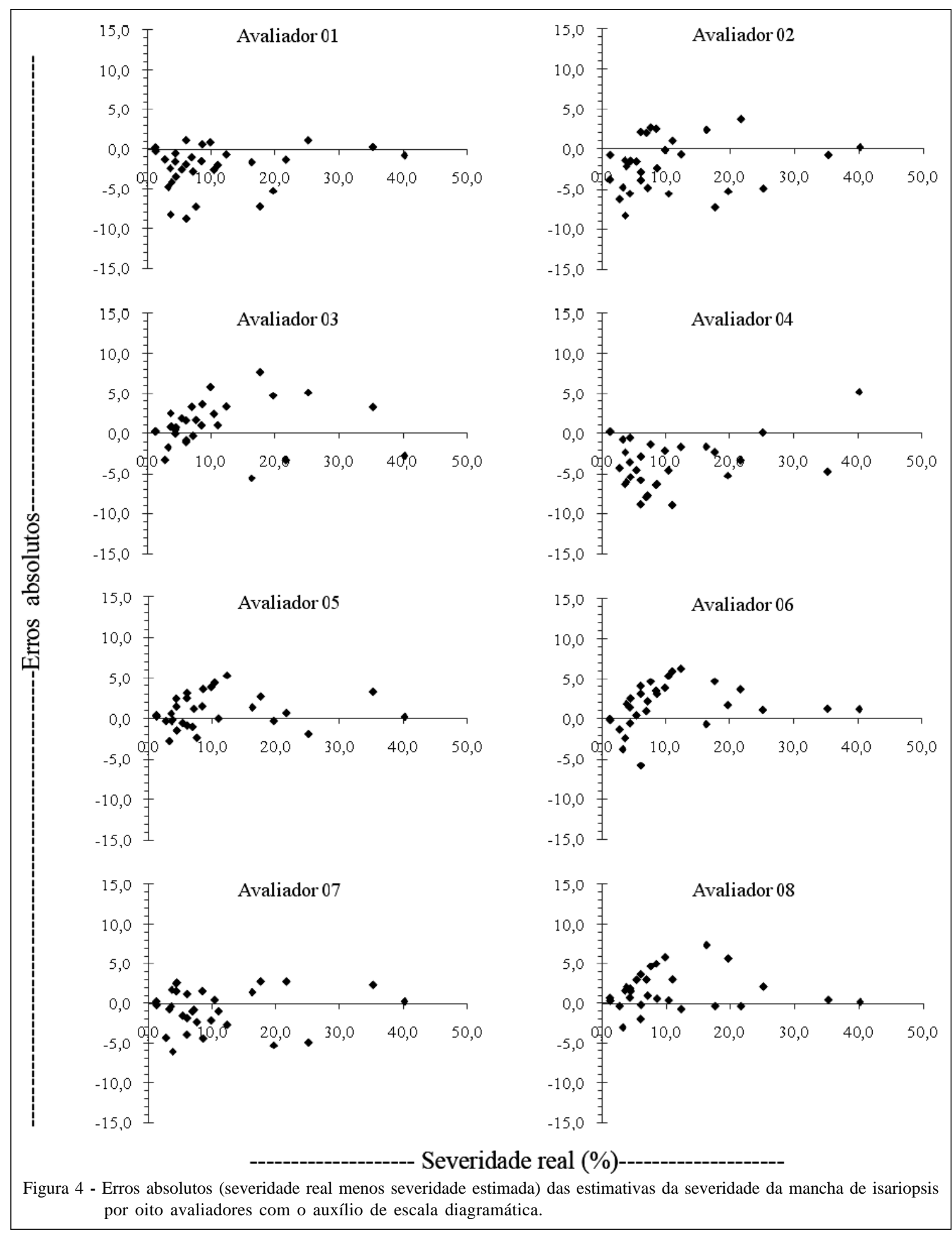

Essa menor variabilidade das estimativas com o uso da escala reforça a reprodutibilidade das estimativas de severidade da doença quando se utilizou a escala diagramática. Isso corrobora a opinião de SPOSITO et al. (2004), os quais afirmam que a precisão e a acurácia das estimativas da severidade aumentam quando a avaliação é realizada com escalas diagramáticas, em comparação com avaliações realizadas sem o auxílio dessas ferramentas.

Na primeira etapa de validação da escala diagramática, ou seja, sem a sua utilização, os valores do intercepto de todos os avaliadores diferiram 
Tabela 1 - Acurácia e precisão representadas pela interseção $(a)$, pela inclinação $(b)$ e pelo coeficiente de determinação $\left(R^{2}\right)$ nas avaliações da severidade da mancha de isariopsis da videira, causada por Isariopsis clavispora, sem e com o auxílio da escala diagramática ( $y=$ $a+b x$, em que $y$ representa a severidade estimada e $x$, a severidade real).

\begin{tabular}{|c|c|c|c|c|c|c|}
\hline \multirow{2}{*}{ Avaliador } & \multicolumn{3}{|c|}{ 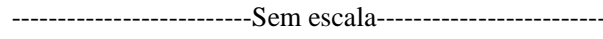 } & \multicolumn{3}{|c|}{---Com escala------ } \\
\hline & $a^{(\mathrm{i})}$ & $b^{(i i)}$ & $R^{2}(\%)$ & $a$ & $b$ & $R^{2}(\%)$ \\
\hline 01 & $0,84^{*}$ & $0,68^{\mathrm{ns}}$ & 0,65 & $-1,91^{\mathrm{ns}}$ & $0,97^{\mathrm{ns}}$ & 0,92 \\
\hline 02 & $3,56^{*}$ & $0,26^{\mathrm{ns}}$ & 0,36 & $-1,27^{*}$ & $0,95^{\mathrm{ns}}$ & 0,91 \\
\hline 03 & $2,08^{*}$ & $0,63^{\mathrm{ns}}$ & 0,60 & $1,72^{\mathrm{ns}}$ & $0,94^{\mathrm{ns}}$ & 0,91 \\
\hline 04 & $0,20^{*}$ & $0,45^{\mathrm{ns}}$ & 0,60 & $-3,67^{\mathrm{ns}}$ & $1,00^{\mathrm{ns}}$ & 0,89 \\
\hline 05 & $1,38^{*}$ & $0,51^{\mathrm{ns}}$ & 0,70 & $1,09^{*}$ & $0,98^{\mathrm{ns}}$ & 0,96 \\
\hline 06 & $2,36^{*}$ & $0,53^{\mathrm{ns}}$ & 0,34 & $1,81^{\mathrm{ns}}$ & $0,97^{\mathrm{ns}}$ & 0,91 \\
\hline 07 & $2,67^{*}$ & $0,68^{\mathrm{ns}}$ & 0,37 & $0,24^{*}$ & $0,96^{\mathrm{ns}}$ & 0,93 \\
\hline 08 & $-0,79^{*}$ & $0,99^{\mathrm{ns}}$ & 0,73 & $2,11^{\mathrm{ns}}$ & $0,95^{\mathrm{ns}}$ & 0,94 \\
\hline Média & 1,73 & 0,59 & 0,54 & 1,52 & 0,96 & 0,92 \\
\hline
\end{tabular}

${ }^{\text {(i) }}$ asterisco indica que o valor da interseção da reta ( $a$ ) foi diferente de zero pelo teste $t(P=0,05)$, ${ }^{\text {ns }}$ indica que não houve diferença estatística significativa entre $a$ e zero pelo teste $t(P=0,05)$; ${ }^{\text {(ii) }}$ asterisco indica que o valor do coeficiente angular da reta de regressão $(b)$ foi diferente de um pelo teste $t(P=0,05)$; ns indica que não houve diferença estatística significativa entre $b$ e um pelo teste $t(P=0,05)$.

significativamente de zero para as retas de regressão entre a severidade real e a estimada (Tabela 1 ). Na média dos avaliadores, o valor do intercepto e do coeficiente angular foi de 1,73 e 0,59 , respectivamente. Com a utilização da escala, os valores do intercepto de $37,5 \%$ dos avaliadores (02, 05, e 07) diferiram significativamente de zero. Todos os valores do coeficiente angular da reta dos avaliadores não diferiram significativamente de um, tanto com, quanto sem a utilização da escala diagramática.

Houve redução nos erros absolutos para as estimativas com auxílio da escala, quando comparada com a distribuição dos resíduos das estimativas obtidas sem escala diagramática. Os erros absolutos observados para as estimativas obtidas por todos os avaliadores, com o auxílio da escala, ficaram dentro de valores aceitáveis $(-10$ a $+10 \%)$, segundo critérios adotados por programas de treinamento na quantificação de doenças, como o Distrain ${ }^{\circledR}$ (TOMERLIN \& HOWELL, 1988) e o Disease Pro ${ }^{\circledR}$ (NUTTER \& WORAWITLIKIT, 1989).

A distribuição dos resíduos das avaliações realizadas sem o auxílio da escala diagramática apresentou estimativas com erros absolutos variando entre -68,29 (Figura 3, avaliador 02) e 15,74 (Figura 3, avaliador 07), sendo a média dos erros (em módulo) de todos os avaliadores igual a 6,34. Quando a avaliação foi realizada com o auxílio da escala, todos os avaliadores melhoraram sua precisão e reduziram os erros sistemáticos das estimativas (Figura 4), sendo a média dos erros (em módulo) de todos os avaliadores igual a 0,42 .
A adoção dessa escala em estudos epidemiológicos poderá reduzir a subjetividade das estimativas e propiciar informações mais precisas a respeito do patossistema Isariopsis clavispora em videira, além de proporcionar uma padronização das avaliações de severidade, permitindo, assim, a comparação de resultados entre diferentes instituições e locais.

\section{CONCLUSÕES}

Os níveis de acurácia, precisão e reprodutibilidade das mensurações da severidade da mancha de isariopsis da videira melhoram com o uso da escala diagramática.

\section{REFERÊNCIAS}

AMORIM, L. Avaliação de doenças. In: BERGAMIN FILHO, A. et al (Eds.). Manual de fitopatologia. São Paulo: Ceres, 1995. V.1, p.647-671.

AMORIM, L.; KUNIYUKI, H. Doenças da videira. In: AMORIM, H.K et al. (Eds.). Manual de fitopatologia. São Paulo: Ceres, 1997. V.2, p.736-757.

AQUINO, L.A. et al. Elaboração e validação de escala diagramática para quantificação da mancha de ramularia do algodoeiro. Summa Phytopathologica, v.34, n.4, p.361-363, 2008. Disponível em: <http://www.scielo.br/scielo.php?script=sci_arttext\&pid=S010054052008000400012\&lng=pt\&nrm=i\&tlng=pt $>$. Acesso em: 01 jul. 2009. doi: 10.1590/S0100-54052008000400012.

BERGAMIN FILHO, A.; AMORIM, L. Doenças de plantas tropicais: epidemiologia e controle econômico. São Paulo: Agronômica Ceres, 1996. 299p. 
GODOY, C.V. et al. Diagrammatic scale for assessment of soybean rust severity. Fitopatologia Brasileira, v.31, n.1, p.63-68, 2006. Disponível em: <http://www.scielo.br/ s ci e lo.ph p ? s c ri pt = sci_art text \& pid = S $0100-$ 41582006000100011>. Acesso em: 01 jul. 2009. doi: 10.1590/ S0100-41582006000100011.

GODOY, C.V. et al. Diagrammatic scales for bean diseases: Development and validation. Journal of Plant Disease and Protection, v.104, n.4, p.336-345, 1997.

GOMES, A.M.A. et al. Elaboração e validação de escala diagramática para cercosporiose da alface. Summa Phytopathologica, v.30, n.1, p.38-42, 2004.

HORSFALL, J.C.; BARRATT R.W. An improved grading system for measuring plant diseases. Phytopathology, v.35, p.665, 1945.

HORSFAlL, J.G.; COWLING, E.B. (Ed.). Plant disease: an advanced treatise - how disease develops in populations. New York: Academic, 1978. V.2, p.119-136.

LENZ, G. et al. Escala diagramática para avaliação de severidade de mancha-de-septoria em girassol. Ciência Rural, ahead of print, 2009.

MARTINS, M.C. et al. Escala diagramática para quantificação do complexo de doenças foliares de final de ciclo em soja. Fitopatologia Brasileira, v.29, n.2, p.179-184, 2004. Disponível em: <http://www.scielo.br/scielo.php?pid=S010041582004000200009\&script=sci_arttext\&tlng=es $>$. Acesso em: 01 jul. 2009. doi: 10.1590/S0100-41582004000200009.

NASCIMENTO, A.R.P. et al. Elaboração e validação de escala diagramática para cancro bacteriano da videira. Summa Phytopathologica, v.31, n.1, p.59-64, 2005.
NUTTER JR., F.W.; SCHULTZ, P.M. Improving the accuracy and precision of disease assessments: selection of methods and use of computer-aided training programs. Canadian Journal of Plant Pathology, v.17, n.1, p.174-184, 1995.

NUTTER JR., F.W.; WORAWITLIKIT, O. Disease Pro: a computer program for evaluating and improving a person's ability to assess disease proportion. Phytopathology, v.79, p.1135, 1989.

SONEGO, O.R.; GARRIDO, L.R. Uvas americanas e híbridas para processamento em clima temperado - Doenças fúngicas e medidas de controle. Sistema de Produção, v.2, p.25, 2003.

SPOSITO, M.B. et al. Elaboração e validação de escala diagramática para avaliação da severidade da mancha preta em frutos cítricos. Fitopatologia Brasileira, v.29, n.1, p.81-85, 2004. Disponível em: <http://www.scielo.br/scielo.php?script=sci_arttext\&pid=S010041582004000100012>. Acesso em: 01 jul. 2009. doi: 10.1590/ S0100-41582004000100012.

STONEHOUSE, J. Assessment of Andean bean disease using visual keys. Plant Pathology, v.43, n.4, p.519-527, 1994.

TOMERLIN, JR.; HOWELL, T.A. Distrain: a computer program for training people to estimate disease severity on cereal leaves. Plant Disease, v.72, p.455-459, 1988.

TOVAR-SOTO, A. et al. Escala logarítmica diagramática de severidad de la mancha negra (Colletotrichum gloeosporioides Penz.) en Chirimoyo (Annona cherimola Mill). Revista Mexicana de Fitopatología, v.20, n.1, p.103-109, 2002.

ZEMOLIN, C.R. et al. Mancha das folhas da videira (Isariopsis clavispora $=$ Pseudocercospora vitis). Santa Maria: UFSM, 2006. (Informe técnico n. 12/2006). 